\title{
Radical cystectomy-what is the optimal surgical approach?
}

\author{
Gregory J. Nason, Khaled Ajib, Guan-Hee Tan, Girish S. Kulkarni \\ Division of Urology, Departments of Surgery and Surgical Oncology, Princess Margaret Cancer Centre, Toronto, ON, Canada \\ Correspondence to: Girish S. Kulkarni, MD PhD FRCSC. Staff Urologist, Princess Margaret Cancer Centre, Associate Professor, Department of \\ Surgery (Urology), University of Toronto, 700 University Avenue - Suite - 6-824, Toronto, Ontario, Canada. Email: girish.kulkarni@uhn.ca. \\ Comment on: Khan MS, Omar K, Ahmed K, et al. Long-term Oncological Outcomes from an Early Phase Randomised Controlled Three-arm Trial \\ of Open, Robotic, and Laparoscopic Radical Cystectomy (CORAL). Eur Urol 2020;77:110-8.
}

Submitted Apr 23, 2020. Accepted for publication May 29, 2020.

doi: $10.21037 /$ tau-20-880

View this article at: http://dx.doi.org/10.21037/tau-20-880

Bladder cancer is the $9^{\text {th }}$ most common cancer in the world and $4^{\text {th }}$ most common cancer diagnosed in men in the United States (1). The age-standardized incidence rate (per 100,000 person/years) is 9.0 for men and 2.2 for women (2).

Approximately $30 \%$ of patients diagnosed with nonmuscle invasive bladder cancer (NMIBC) progress to muscle invasive bladder cancer (MIBC), while a further $25 \%$ present upfront as MIBC (1). The treatment options are based upon stage at diagnosis. The current standard of care for the treatment of MIBC is radical cystectomy (3-5). Bladder sparing approaches are an alternative for patients with MIBC who are too comorbid to undergo a radical cystectomy or who refuse a radical. The guidelines regarding MIBC from the urological societies [European Association of Urology (EAU), American Urological Association (AUA), American Society of Clinical Oncology (ASCO), American Society of Radiation Oncology (ASTRO), Society of Urologic Oncology (SUO) and National Comprehensive Cancer Network (NCCN)] recommend radical cystectomy and pelvic lymph node dissection as the gold standard treatment for patients with MIBC. There is also evidence supporting the use of cisplatin-based neoadjuvant chemotherapy (3-5).

A significant proportion of MIBC patients still develop metastatic disease despite stage appropriate treatment with radical cystectomy and neo-adjuvant chemotherapy (3). The 5 -year overall survival with MIBC is only $50 \%$ despite treatment (6). Untreated patients do very poorly with symptomatic local progression, development of metastases and mortality. Fewer than $15 \%$ survive longer than 2 years if untreated (1).

In the article entitled 'Long-term Oncological Outcomes from an Early Phase Randomised Controlled Threearm Trial of Open, Robotic, and Laparoscopic Radical Cystectomy (CORAL)' published in European Urology in January 2020, the bladder group from Guy's and St Thomas' Hospital in London report the oncological outcomes of a randomized control trial (RCT) of radical cystectomy operative technique (7). The CORAL trial was a three arm RCT comparing open, laparoscopic and robotic radical cystectomy. With over 5 years of follow-up, 60 patients were randomized with primary end points of recurrence free survival (RFS), cancer specific survival (CSS) and overall survival (OS). The authors concluded robotic and laparoscopic radical cystectomy achieved equivalent oncological outcomes to the gold standard of open radical cystectomy. The 5-year RFS was $60 \%, 58 \%$, and $71 \%$; 5 -year CSS was $64 \%, 68 \%$, and $69 \%$; and 5 -year OS was $55 \%, 65 \%$, and $61 \%$ for open, robotic, and laparoscopic radical cystectomy, respectively. There was no significant survival differences noted based on surgical modality.

The durability of open radical cystectomy is well known from numerous large retrospective studies. The goals in the management of MIBC include oncological and functional outcomes as well as quality of life. Any move to a minimally invasive approach needs to at the very least match that of open cystectomy.

The 5 and 10-year OS rates associated with open radical cystectomy have been reported around $60-66 \%$ and $43 \%$, respectively $(8,9)$, which are comparable to the 5 -year OS results of the CORAL trial. Furthermore, similar to the findings of the CORAL Trial, an open series by Madersbacher et al., revealed a high rate of undiagnosed nodal metastases $(24 \%)$ which were associated with poor 
long term survival (9). Radical cystectomy ensured a low pelvic recurrence rate even in lymph node-positive patients.

Open radical cystectomy is a morbid procedure with complication rates reported between $30 \%$ and $70 \%$, even at high volume centers (10-12). Any effort to reduce the morbidity associated with the procedure should be welcomed.

Along these lines, although the traditional approach to radical cystectomy has been an open operation, minimally invasive approaches with the promise of decreased complications and improved quality of life have evolved over time. Ten-year outcomes have been reported by the International Robotic Cystectomy Consortium with longterm oncologic outcomes, recurrence rates and recurrence patterns after robotic cystectomy seemingly comparable to those in open series (13). At a median follow-up of 5 years, local and distant recurrence had developed in 15\% and 29\% of patients, respectively. Ten-year RFS, CSS and OS rates were $59 \%, 65 \%$ and $35 \%$, respectively.

Robotic surgery has become a mainstay of practice in urology in recent times. Despite a lack of level one evidence supporting robotic prostatectomy (14), the majority of prostatectomies are performed robotically in the US. As confidence with robotic surgery increases, the indications have expanded. The advantages of robotic surgery are well documented including less blood loss, shorter length of stay, decreased analgesia requirements and quicker return to daily activities. Increasing surgeon skill and confidence have also extended the application of robotics to radical cystectomy. The CORAL trial represents one of the RCTs assessing the role of robotic cystectomy and laparoscopic cystectomy (minimally invasive cystectomy).

Opponents of robotic (and laparoscopic) cystectomy have questioned whether the quality of an MIS approach can mirror that of an open operation. To address this concern, surgical margin status and nodal yield have been scrutinized as surrogates of surgical technique. No difference was noted in positive margins between the CORAL groups: $10 \%$ (open), 15\% (robotic), $5 \%$ (laparoscopic). Mean nodal yield was $18.8,16.3$, and 15.5 , respectively, with the difference only reaching statistical significance between the open and laparoscopic arms.

Another larger RCT comparing open versus robotic cystectomy was the RAZOR trial which reported in the Lancet in 2018 (15). That landmark paper demonstrated that robotic cystectomy was non-inferior to open cystectomy for 2-year progression free survival (PFS). The RAZOR trial recently reported updated results in the fournal of Urology in January 2020 (16). Specifically, the 3-year PFS was 68.4\% (95\% CI: 60.1-75.3\%) and 65.4\% (95\% CI: 56.8-72.7\%) in the robotic and open groups, respectively $(\mathrm{P}=0.600)$. The 3 -year OS was $73.9 \%$ (95\% CI: $65.5-80.5 \%$ ) and $68.5 \%$ (95\% CI: $59.8-75.7 \%$ ) in the robotic and open groups, respectively $(\mathrm{P}=0.334)$. Although 3 -year survival data were not explicitly reported in the CORAL trial an examination of the Kaplan-Meier curves at the 3-year mark suggests that the OS for both open and robotic cystectomy lies between $60 \%$ and $80 \%$ which are comparable to the RAZOR trial.

There are a number of key differences between the RAZOR and CORAL trials. First, the CORAL trial was a single site study comparing open, robotic and laparoscopic surgery whereas RAZOR was a multi-centre study across 15 sites in the US comparing open and robotic surgery. Second, the RAZOR trial was much larger involving 302 patients compared with only 60 in the CORAL trial. Third, the RAZOR trial was actually performed as a non-inferiority study with a priori defined endpoints for non-inferiority whereas the CORAL trial failed to accrue to completion and did not specify in the methodology a non-inferiority boundary, thus diminishing the veracity of the conclusion of "equivalence" across the three treatment modalities.

Nevertheless, the CORAL trial serves as a potential landmark paper for robotic cystectomy. It supports the oncological efficacy of robotic surgery in experienced hands with short and long term data comparable across treatment groups. Minimally invasive techniques simply represent a surgical approach to an operation; thus, it should not matter how a procedure is performed so long as it is done safely with acceptable oncological and functional results. Data from open versus robotic RCTs, suggest that surgeons should perform radical cystectomy using whichever technique they are most comfortable with as opposed to being focused on an open or minimally invasive approach. Nevertheless, there have been some doubters of the robotic platform with concerns regarding unusual recurrence patterns. Bochner et al., while reporting no difference in the secondary outcomes (RFS, CSS, OS) of their safety RCT comparing open versus robotic cystectomy in 118 patients at Memorial Sloan Kettering (17), did raise the concern regarding patterns of first recurrence associated with the robotic approach with a greater number of local/ abdominal sites (hazard ratio: 0.34; 95\% CI: 0.12-0.93; $\mathrm{P}=0.035)$. The renowned testis cancer group from Indiana University expressed similar concerns regarding recurrence 
patterns with robotic RPLND in a small case series (18). Similarly, unusual port site metastases have been reported after robotic renal surgery (19). Furthermore, two landmark studies in the field of gynecologic oncology reporting worse survival outcomes associated with the minimally invasive approaches (20,21). All of these findings have led some to question the true oncologic safety of intra-abdominal pneumoperitoneum and robotic surgery in general.

To address the concerns of unusual recurrence patterns, the CORAL trial specifically analyzed each event in the $39 \%$ of patients who developed a recurrence. Recurrences were classified as local, distant or a combination of both. Both open and robotic cystectomy had 3 local recurrences and 4 distant recurrences. Surgical approach was not deemed to effect recurrence (HR 1.13; 95\% CI: 0.42-3.01), overall death (HR 0.72; 95\% CI: $0.30-1.74$ ), or bladder cancerspecific death (HR 0.75;95\% CI: 0.28-2.01). Similarly, the RAZOR trial showed no difference in recurrence patterns (HR 0.95; 95\% CI: 0.61-1.47, $\mathrm{P}=0.805$ ).

One limitation of the CORAL trial is that the radical cystectomy was performed by 3 expert surgeons with significant experience in minimally invasive cystectomy who were felt to be beyond their learning curve. Thus, the success of the CORAL trial may not be generalisable outside of a high volume centre. Further limitations of the trial include a lack of quality of life data and a cost analysis. The RAZOR trial reported no difference in health-related quality of life between the surgical approaches at 3 or 6 months. They did note however that emotional wellbeing increased from baseline in both arms over time (22). Given that the oncological outcomes are no different- if we are to pursue a more costly alternative (robotic radical cystectomy) then one surmises that there needs to be some measurable gain.

The authors of the CORAL trial should be commended for completing the daunting task of a surgical RCT. Readers should take note however that this small trial was conducted by an expert bladder centre with all three surgeons well beyond their robotic learning curve. Furthermore, the reconstructive component of the radical cystectomy was performed extra-corporeally. It may be possible that a completely intra-corporeal reconstruction can lead to variations in oncological or quality of life outcomes. Thus, a further challenge may be to compare intra- versus extracorporeal reconstruction. Although this is unlikely to have any effect regarding oncological control, the reconstructive portion of the procedure is where many of the peri- and post-operative complications originate. It is thus fair to hypothesize that an intra-corporeal reconstruction leads to diminished complications and an improved quality of life. The iROC study, a prospective multicentre randomised controlled trial comparing the outcomes from robotic radical cystectomy with intracorporeal reconstruction with open radical cystectomy, addresses this objective. The study aims to accrue 340 patients by the end of 2020 and assesses 90-day complications and 1-year quality of life outcomes (23).

There have been a number of recent meta-analyses comparing open and robotic radical cystectomy; summarily, a robotic approach has not been shown to have a considerable impact on oncological outcomes, complication rates and quality of life outcomes compared to open cystectomy $(24,25)$. The benefits of robotic radical cystectomy are a reduction in blood transfusion rates and shorter length of stay. The results of the CORAL trial substantiate this; we believe this trial has contributed to the growing evidence of non-inferiority of robotic cystectomy compared to open cystectomy in experienced hands.

\section{Acknowledgments}

Funding: None.

\section{Footnote}

Provenance and Peer review: This article was commissioned by the editorial office, Translational Andrology and Urology. The article did not undergo external peer review.

Conflicts of Interest: All authors have completed the ICMJE uniform disclosure form (available at http://dx.doi. org/10.21037/tau-20-880). The authors have no conflicts of interest to declare.

Ethical Statement: The authors are accountable for all aspects of the work in ensuring that questions related to the accuracy or integrity of any part of the work are appropriately investigated and resolved.

Open Access Statement: This is an Open Access article distributed in accordance with the Creative Commons Attribution-NonCommercial-NoDerivs 4.0 International License (CC BY-NC-ND 4.0), which permits the noncommercial replication and distribution of the article with 
the strict proviso that no changes or edits are made and the original work is properly cited (including links to both the formal publication through the relevant DOI and the license). See: https://creativecommons.org/licenses/by-nc-nd/4.0/.

\section{References}

1. Antoni S, Ferlay J, Soerjomataram I, et al. Bladder Cancer Incidence and Mortality: A Global Overview and Recent Trends. Eur Urol 2017;71:96-108.

2. Ferlay J, Soerjomataram I, Dikshit R, et al. Cancer incidence and mortality worldwide: sources, methods and major patterns in GLOBOCAN 2012. Int J Cancer 2015;136:E359-86.

3. Alfred Witjes J, Lebret T, Compérat EM, et al. Updated 2016 EAU Guidelines on Muscle-invasive and Metastatic Bladder Cancer. Eur Urol 2017;71:462-75.

4. Chang SS, Bochner BH, Chou R, et al. Treatment of NonMetastatic Muscle-Invasive Bladder Cancer: AUA/ASCO/ ASTRO/SUO Guideline. J Urol 2017;198:552-9.

5. Spiess PE, Agarwal N, Bangs R, et al. Bladder Cancer, Version 5.2017, NCCN Clinical Practice Guidelines in Oncology. J Natl Compr Canc Netw 2017;15:1240-67.

6. Martini A, Sfakianos JP, Renström-Koskela L, et al. The natural history of untreated muscle-invasive bladder cancer. BJU Int 2020;125:270-5.

7. Khan MS, Omar K, Ahmed K, et al. Long-term Oncological Outcomes from an Early Phase Randomised Controlled Three-arm Trial of Open, Robotic, and Laparoscopic Radical Cystectomy (CORAL). Eur Urol 2020;77:110-8.

8. Stein JP, Lieskovsky G, Cote R, et al. Radical cystectomy in the treatment of invasive bladder cancer: long-term results in 1,054 patients. J Clin Oncol 2001;19:666-75.

9. Madersbacher S, Hochreiter W, Burkhard F, et al. Radical cystectomy for bladder cancer today--a homogeneous series without neoadjuvant therapy. J Clin Oncol 2003;21:690-6.

10. Shabsigh A, Korets R, Vora KC, et al. Defining early morbidity of radical cystectomy for patients with bladder cancer using a standardized reporting methodology. Eur Urol 2009;55:164-74.

11. Svatek RS, Fisher MB, Matin SF, et al. Risk factor analysis in a contemporary cystectomy cohort using standardized reporting methodology and adverse event criteria. J Urol 2010;183:929-34.

12. Hautmann RE, de Petriconi RC, Volkmer BG. Lessons learned from 1,000 neobladders: the 90-day complication rate. J Urol 2010;184:990-4.

13. Hussein AA, Elsayed AS, Aldhaam NA, et al. Ten-Year Oncologic Outcomes Following Robot-Assisted Radical Cystectomy: Results from the International Robotic Cystectomy Consortium. J Urol 2019;202:927-35.

14. Coughlin GD, Yaxley JW, Chambers SK, et al. Robotassisted laparoscopic prostatectomy versus open radical retropubic prostatectomy: 24-month outcomes from a randomised controlled study. Lancet Oncol 2018;19:1051-60.

15. Parekh DJ, Reis IM, Castle EP, et al. Robot-assisted radical cystectomy versus open radical cystectomy in patients with bladder cancer (RAZOR): an open-label, randomised, phase 3, non-inferiority trial. Lancet 2018;391:2525-36.

16. Venkatramani V, Reis IM, Castle EP, et al. Predictors of Recurrence, and Progression-Free and Overall Survival following Open versus Robotic Radical Cystectomy: Analysis from the RAZOR Trial with a 3-Year Followup. J Urol 2020;203:522-9.

17. Bochner BH, Dalbagni G, Marzouk KH, et al. Randomized Trial Comparing Open Radical Cystectomy and Robot-assisted Laparoscopic Radical Cystectomy: Oncologic Outcomes. Eur Urol 2018;74:465-71.

18. Calaway AC, Einhorn LH, Masterson TA, et al. Adverse Surgical Outcomes Associated with Robotic Retroperitoneal Lymph Node Dissection Among Patients with Testicular Cancer. Eur Urol 2019;76:607-9.

19. Song J, Kim E, Mobley J, et al. Port site metastasis after surgery for renal cell carcinoma: harbinger of future metastasis. J Urol 2014;192:364-8.

20. Ramirez PT, Frumovitz M, Pareja R, et al. Minimally Invasive versus Abdominal Radical Hysterectomy for Cervical Cancer. N Engl J Med 2018;379:1895-904.

21. Melamed A, Margul DJ, Chen L, et al. Survival after Minimally Invasive Radical Hysterectomy for Early-Stage Cervical Cancer. N Engl J Med 2018;379:1905-14.

22. Becerra MF, Venkatramani V, Reis IM, et al. HealthRelated Quality of Life of Patients with Bladder Cancer in the RAZOR Trial - A Multi-Institutional Randomized Trial Comparing Robot versus Open Radical Cystectomy. J Urol 2020;204:450-9.

23. Trial to Compare Robotically Assisted Radical Cystectomy With Open Radical Cystectomy (iROC). Available online: https://clinicaltrials.gov/ct2/show/NCT03049410 (Accessed 22th April 2020)

24. Satkunasivam R, Tallman CT, Taylor JM, et al. Robot- 
assisted Radical Cystectomy Versus Open Radical Cystectomy: A Meta-analysis of Oncologic, Perioperative, and Complication-related outcomes. Eur Urol Oncol 2019;2:443-7.

Cite this article as: Nason GJ, Ajib K, Tan GH, Kulkarni GS. Radical cystectomy-what is the optimal surgical approach? Transl Androl Urol 2020;9(5):2308-2312. doi:10.21037/tau-20-880
25. Sathianathen NJ, Kalapara A, Frydenberg M, et al. Robotic Assisted Radical Cystectomy vs Open Radical Cystectomy: Systematic Review and Meta-Analysis. J Urol 2019;201:715-20. 